\title{
Three Arguments from Temporary Intrinsics
}

\author{
Philosophy \& Phenomenological Research (2010) 81: 605-619
}

\begin{abstract}
The Argument from Temporary Intrinsics is one of the canonical arguments against endurantism. I show that the two standard ways of presenting the argument have limited force. I then present a new version of the argument, which provides a more promising articulation of the underlying objection to endurantism. However, the premises of this argument conflict with the gauge theories of particle physics, and so this version of the argument is no more successful than its predecessors. I conclude that no version of the Argument from Temporary Intrinsics gives us a compelling reason to favor one theory of persistence over another.
\end{abstract}

\section{The Problem}

I am bent at one time and straight at another. But I cannot be both bent and straight, since then I would instantiate contradictory properties. So what underwrites this change?

Prima facie, an object undergoes change whenever it gains or loses a certain kind of property. These properties are often called temporary intrinsics. The term "temporary intrinsics" can be misleading, however, since whether such properties are intrinsic is one of the questions at issue. To avoid confusion, I will use the term ephemera to refer to those properties and relations involved in ordinary matters of change.

Two popular theories of persistence - perdurantism and endurantism provide different accounts of change. According to the perdurantist, objects have temporal parts, each of which may instantiate different properties. Change occurs whenever one temporal part has an ephemeron that another temporal part lacks. In the case above: one of my temporal parts instantiates being bent and another instantiates being straight. Since different temporal parts of me instantiate different ephemera, I undergo change.

The endurantist offers a different response. According to her, ordinary objects do not have temporal parts. Objects endure: they are wholly present at every time at which they exist. How can an object - the whole of it - instantiate being bent as well as the being straight? The standard endurantist response: by instantiating these properties at different times. ${ }^{1}$ For the endurantist, an object's ephemera are two-place relations that hold between objects and times. In the case

\footnotetext{
${ }^{1}$ There are other endurantist responses, some of which I consider in section (2.1).
} 
above: I instantiate being bent relative to time $t_{1}$ and being straight relative to time $t_{2}$. Since I am bent at $t_{1}$ and straight at $t_{2}$, I undergo change. ${ }^{2}$

David Lewis has famously argued that this result constitutes a reductio ad absurdum of endurantism. ${ }^{3}$ When an object undergoes change, says Lewis, this has to do with how the object is in itself and not how it is relative to anything else. But this isn't the case on the endurantist's account. According to endurantism, an object's change has to do with its relations to something else - a time. Since the endurantist fails to respect the observation that an object's change does not involve anything besides the object itself, endurantism is not a tenable position. This argument is known as the Argument from Temporary Intrinsics.

In response to the Argument from Temporary Intrinsics, endurantists have constructed a number of ways to make their account of change, and hence their account of ephemera, more intuitively acceptable. And, in response to these moves, Lewis has proposed a more sophisticated version of the Argument from Temporary Intrinsics. I show that neither the standard argument nor the more sophisticated version has much force.

But that is not the end of the story. There are other ways to understand the Argument from Temporary Intrinsics. The most promising way, I believe, is to understand it as an argument concerning the structure of fundamental properties. I will argue, however, that the premises of this argument conflict with the gauge theories of fundamental physics, and so this version is no more successful than its predecessors. I conclude that no version of the Argument from Temporary Intrinsics gives us a compelling reason to favor one theory of persistence over another.

\section{The Argument from Temporary Intrinsics}

The standard formulation of the perdurantist's objection begins with the notion of intrinsicality. Intuitively, ephemera are intrinsic properties, or properties that an object has in virtue of itself alone. To motivate Lewis's objection to endurantism, let's begin with his characterization of an intrinsic property: a property is intrinsic iff it never differs between duplicates. Two things are duplicates iff they share all their fundamental - or perfectly natural - properties, and their parts can be put into correspondence in such a way that corresponding parts have the same

\footnotetext{
${ }^{2}$ In this paper, I assume that the truth about the world can be stated in a timeless language, and so I will not engage with those who take tense as fundamental.

${ }^{3}$ See Lewis $(1986,202-204)$.
} 
perfectly natural properties and stand in the same perfectly natural relations. ${ }^{4} \mathrm{I}$ may have a duplicate that is five feet from a dog, or has a nephew, or differs in any number of extrinsic ways from myself. But no duplicate of me may differ in sex, lack an appendix, or have an extra limb.

Now we can flesh out Lewis's objection to the endurantist. Suppose I am bent at time $t_{1}$. According to the perdurantist, this consists in my having a bent temporal part that exists at $t_{1}$. Any duplicate of that temporal part likewise instantiates being bent. Since the property being bent never varies between duplicates, it is intrinsic.

Not so for the endurantist, says Lewis. If I am bent at $t_{1}$, then I instantiate the property being bent at $t_{1}$ - a property I have in virtue of bearing the being bent relation to $t_{1 .}{ }^{5}$ But considered by myself, apart from the relations I bear to anything entirely distinct from me, I have no shape at all. ${ }^{6}$ If I have no shape, then no duplicate of me has any shape either; my bentness or straightness is ultimately an extrinsic matter. ${ }^{7}$ Thus, when I change from being bent at $t_{1}$ to being straight at $t_{2}$, the ephemera underlying this change in my shape are not intrinsic. Likewise for all ephemera: if endurantism is true, none of my ephemera are intrinsic.

This is the original Argument from Temporary Intrinsics:

P1. Ephemera are intrinsic.

P2. If endurantism is true, then ephemera are not intrinsic.

C. Endurantism is false.

\subsection{Endurantist Replies}

There are a number of replies available to the endurantist. A natural option is to simply reject Lewis's characterization of intrinsic properties. How can we modify Lewis's characterization to make it amenable to the endurantist? Let us take endurantist duplication, or duplication ${ }_{E}$, to be a four-place relation that holds

\footnotetext{
${ }^{4}$ See Lewis (1983) and (1986, 61-62). Here I assume that the quantifiers are possibilist.

${ }^{5} \mathrm{I}$ am assuming here that the endurantist is taking relations such as being bent as fundamental. Suppose instead that the endurantist takes monadic properties such as being bent at $t_{1}$ as fundamental. If duplicates share all their fundamental properties, then no object will have a duplicate located at another time. If so, then the endurantist's problem is not that intuitively intrinsic properties are rendered extrinsic, but that intuitively extrinsic properties are rendered intrinsic. But the underlying point is the same: the endurantist draws the intrinsic/extrinsic distinction in the wrong place.

${ }^{6}$ See Lewis $(1986,204)$.

${ }^{7}$ Of course, Lewis's characterization of duplication makes use of an atemporal notion of parthood, while most endurantists understand parthood as temporally relativized. I discuss endurantist alternatives to Lewis's approach in section (2.1).
} 
between an object at one time and another object at another time. We can then define intrinsic in the following way:

A property $P$ is intrinsic iff for all $x$ and $y$, and any times $t_{1}$ and $t_{2}$, if $x$ at $t_{1}$ is a duplicate $e_{\mathrm{E}}$ of $y$ at $t_{2}$, then either $P(x)$ and $P(y)$, or $\sim P(x)$ and $\sim P(y)$.

A relation to a time $R$ is intrinsic iff for all $x$ and $y$, and any times $t_{1}$ and $t_{2}$, if $x$ at $t_{1}$ is a duplicate $e_{\mathrm{E}}$ of $y$ at $t_{2}$, then either $R\left(x, t_{1}\right)$ and $R\left(y, t_{2}\right)$, or $\sim R\left(x, t_{1}\right)$ and $\sim R\left(y, t_{2}\right){ }^{8}$

Suppose the relation being bent holds between me and $t_{1}$. Any object at $t_{2}$ that is a duplicate $_{\mathrm{E}}$ of me at $t_{1}$ will likewise bear the relation being bent to $t_{2}$. So on this characterization, being bent is intrinsic - as it intuitively should be. ${ }^{9}$

Alternatively, the endurantist may choose to relativize the instantiation relation, rather than relativize the ephemera themselves. The more familiar twoplace instantiation relation is replaced with a three-place relation that holds between objects, properties, and times. If I am bent at $t_{1}$, then the instantiation relation holds between me, being bent, and $t_{1}$. On this picture of the instantiation relation, ephemera such as being bent remain monadic and intrinsic.

Or, the endurantist can follow Haslanger (1989) in making the truthvalues of propositions relative to times. For example, if I am bent at $t_{1}$ and straight at $t_{2}$, then the proposition that I am bent is true at $t_{1}$ and false at $t_{2}$. Again, on this account, ephemera such as being bent are monadic and intrinsic. ${ }^{10}$

But these responses won't satisfy the perdurantist. Motivating the Argument from Temporary Intrinsics is the conviction that an object has its ephemera in and of itself alone. The endurantist may contrive a sense in which her ephemera are intrinsic and monadic, but nonetheless these properties seem unacceptably relational. The intuitive objection still stands, and the perdurantist needs a better argument to capture it.

\section{The Simpliciter Argument}

Enter "having simpliciter," a notion invoked by David Lewis to better capture this intuitive worry about endurantism. ${ }^{11}$ When object $a$ has property $F$ simpliciter, this has nothing to do with anything besides $a$ and $F$ and simple two-place instantiation. According to the perdurantist, the properties involved in intuitively

\footnotetext{
${ }^{8}$ What about relations that don't involve times, or have more than two relata? These relations won't be intrinsic, which is as it should be.

${ }^{9}$ See also Haslanger $(1989,123)$ for an endurantist-friendly characterization of intrinsicality.

${ }^{10}$ See Caplan (2005), Haslanger (1989, 120), Hinchliff (1996), Lewis (1988, 65-66 fn. 1) and (2002), and van Inwagen (1990) for discussion of the latter two moves.
} 
intrinsic matters of change are had simpliciter. But the endurantist relativizes these properties to times (either by turning them into relations or relational properties, or by temporally relativizing the instantiation relation), and so they are not had simpliciter. This, says Lewis, is unacceptable. ${ }^{12}$

Call this the Simpliciter Argument:

P1'. Ephemera are had simpliciter.

$\mathbf{P 2}^{\prime}$. If endurantism is true, then ephemera are not had simpliciter.

$\mathbf{C}^{\prime}$. Endurantism is false.

This argument is meant to capture the ingredient missing from the standard Argument from Temporary Intrinsics. The endurantist may contest certain tendentious glosses on "intrinsic" and may make instantiation a three-place relation, but still her ephemera are not had simpliciter.

\subsection{Paradigmatic Temporary Intrinsics}

Although the endurantist cannot respond in the same way to the Simpliciter Argument as she could to the Temporary Intrinsics Argument, there are other reasons to worry about the tenability of the Simpliciter Argument. ${ }^{13}$

The force of the argument is proportional to the force of our intuitions about the nature of canonical ephemera. As Merricks (1994) says, it just isn't the case "that all of the properties that an object seems to gain or lose are really relations to times or time indexed. A short list of those properties which are not... includes shape, color, size, and mass." (528)

That seems right. But is it? Arguably, none of these paradigmatic ephemera are had simpliciter. Consider mass. Given the advent of relativity, there are two viable notions of mass: rest mass and relativistic mass. Unlike rest mass, relativistic mass is dependent on reference frame: an object that has a relativistic mass of two grams in one frame may have a relativistic mass of two hundred grams in another. So objects don't have relativistic mass simpliciter; they have relativistic mass relative to a reference frame As Field (1973) argues, our ordinary

\footnotetext{
${ }^{11}$ See Lewis (1986, 52-54); also see Lewis (1988) and (2002).

${ }^{12}$ See Lewis $(2002,4)$.

${ }^{13}$ There are other responses to the Simpliciter Argument that I am not considering here. Wasserman (2003) offers several different responses on behalf of the endurantist. In particular, it seems that the perdurantist can no more accommodate our intuitive beliefs about ordinary objects than the endurantist. Both must deny that ordinary objects have their ephemera simpliciter (for the perdurantist, a persisting object may have a part that is bent simpliciter, but it is not itself bent simpliciter). And, as Wasserman shows, just about any move the perdurantist may make in an attempt to mitigate this counterintuitive result is available to the endurantist as well.
} 
use of "mass" does not determinately pick out rest mass as opposed to relativistic mass. If so, then it is hardly obvious whether objects have mass simpliciter; at the very least, the perdurantist's claim that mass is had simpliciter is not beyond dispute. Moreover, on some views of mass, an object's rest mass is nothing more than its relativistic mass relativized to its own frame of reference. In that case, there is no notion of mass according to which it is had simpliciter. ${ }^{14}$

Or consider color. Many philosophers have argued that color properties are irreducibly relational. For instance, Jackson (1998) argues that the color of an object depends on "a certain kind of creature and circumstances of viewing." (95) If so, objects do not have color simpliciter; they have their colors relative to a creature and circumstances of viewing.

Or consider shape and size. The spatial shape and size of an object supervene on the spatial relations between the object's parts. But in a relativistic world, these relations are frame-dependent. An object that is spherical in one reference frame may be ovoid in another; a jug that holds a gallon of liquid in one frame may hold a half-gallon in another. Objects don't have their spatial shapes and sizes simpliciter; they have them relative to a reference frame. ${ }^{15}$

In sum, it's arguably the case that none of the paradigmatic ephemera are had simpliciter, regardless of one's preferred theory of persistence. This doesn't rule out the possibility that some ephemera are had simpliciter (if endurantism is false). But the further we move away from the canonical ephemera, the weaker the argument becomes. The Simpliciter Argument is only as strong as the strength of the intuitions it captures; and while we seem to have firm intuitions about canonical ephemera like shape - "if we know what shape is, we know it is a property, not a relation" (Lewis 1986, 204) - our intuitions about other ephemera are more tenuous. (And it seems that our intuitions about the intrinsicality of shape are rather far off the mark.) At the very least, the tentative nature of the argument gives us motivation to look for something better.

More importantly, the fact that the argument is vulnerable to the sort of piecemeal rebuttal given above suggests that it isn't getting at the real worry. The debate over persistence shouldn't depend, it seems, on a miscellany of unrelated issues. The perdurantist's objection is more general: even if canonical ephemera like color and shape aren't had simpliciter, then surely there is something underlying these properties that is. It is this thought that I aim to capture with the argument I present in section (4). But let's first examine some replies on behalf of

\footnotetext{
${ }^{14}$ I thank an anonymous referee for this point.

${ }^{15}$ Bricker (1993) and Skow (2007) both offer different reasons to think that shape is not intrinsic.
} 
the perdurantist.

\subsection{Two Perdurantist Replies}

I've found in conversation that perdurantists who endorse the Simpliciter Argument tend to raise two responses to the criticisms given above. The first response is to insist that there is a sense in which some of the canonical ephemera - particularly shape and size - are had simpliciter. The second response is to change the desiderata for a satisfactory account of ephemera, and amend the Simpliciter Argument in light of this.

\section{Response 1: Senses of "Shape"}

The perdurantist who offers the first sort of response might claim that, in the context of ephemera, the relevant notion of shape isn't spatial (or three-

dimensional) shape, since spatial shape is not had simpliciter. Rather, the relevant notion of shape is four-dimensional shape. Four-dimensional shape is had simpliciter, says our perdurantist; it does not involve relations to reference frames, times, or anything else. ${ }^{16}$ So the response goes: any theory of persistence must capture the intuition that an object has its four-dimensional shape simpliciter. Since the perdurantist can capture this intuition while the endurantist cannot, perdurantism is to be preferred.

This reply does not work for several reasons. First, our pretheoretic notion of shape is spatial, not spatiotemporal, so the intuitions about having simpliciter that the perdurantist invokes apply to three-dimensional shape, not four-dimensional shape. Now, the perdurantist might be tempted to reply that our intuitions about having simpliciter do apply to four-dimensional shape. After all, the notion of four-dimensional shape isn't so much harder to grasp than that of three-dimensional shape. Most of us understand the relationship between two and three-dimensional shape, and four-dimensional shape can be described analogously: simply increase the number of dimensions by one, and call the newest addition "time." Even students in an introductory philosophy class unfamiliar with the notion of four-dimensional shape have little trouble grasping the concept of a spacetime "worm" or an object's "worldline."

But this line of thought is plausible only if one conflates two different notions of "four-dimensional shape." Call the first notion "Newtonian" and the second "Relativistic." Think of four-dimensional Newtonian shape as the shape of an object as it sweeps through time. Just as a two-dimensional coordinate system

\footnotetext{
${ }^{16}$ Ted Sider has raised this response on behalf of the perdurantist in conversation.
} 
$(x, y)$ can be expanded into a three-dimensional coordinate system $(x, y, z)$ by adding on a $z$ axis, so a three-dimensional coordinate system can be expanded into a four-dimensional Newtonian coordinate system $(x, y, z, t)$ by adding a temporal axis. The four-dimensional Relativistic shape of an object, on the other hand, is the information encoded by the spatiotemporal intervals between each of the object's parts.

I'll grant that four-dimensional Newtonian shape is an intuitively accessible notion of shape. But Newtonian shape isn't had simpliciter. An object's Newtonian shape depends on the spatial distances between its parts, and these facts are frame-dependent. On the other hand, Relativistic shape is had simpliciter. An object's Relativistic shape depends on the spatiotemporal intervals between each of is parts, and these facts are not frame-dependent. But we don't have an intuitive understanding of these spatiotemporal intervals, and a fortiori, we don't have an intuitive understanding of four-dimensional Relativistic shape. (Those who believe our ordinary intuitions about distance apply to these relativistic spatiotemporal intervals should reconsider: unlike spatial intervals, spatiotemporal intervals can be represented by both real and imaginary numbers, and there can be an infinite number of distinct locations separated by a spatiotemporal interval of zero.)

The perdurantist's initial complaint against the endurantist was that she fails to respect the observation that certain properties of everyday acquaintance, such as shape, are had simpliciter. This line of response attempts to extend the complaint to four-dimensional shape, but it does not succeed. If by "shape" the perdurantist means "four-dimensional Newtonian shape," then shape is not had simpliciter. And if by "shape" she means "four-dimensional Relativistic shape," then shape is not a property of everyday acquaintance.

There's another reason that shifting to four-dimensional Relativistic shape does not help the perdurantist. The endurantist was initially faulted for her account of the properties involved when an object undergoes change. But fourdimensional Relativistic shape is not a property involved in matters of change: no object can have one four-dimensional shape at time $t_{1}$ and another at time $t_{2}$. Since Relativistic shape is not a temporal property, there is nothing to stop the endurantist from agreeing that objects have their four-dimensional Relativistic shapes simpliciter.

In sum, both the perdurantist and the endurantist must admit that no object has its spatial extension simpliciter, and both may say that objects have their frame-invariant shapes simpliciter. The strategy of shifting the properties in question to frame-invariant ones does not make the Simpliciter Argument any 
more effective.

\section{Response 2: Time and Having Simpliciter}

The perdurantist who endorses the second sort of response does not attempt to contrive a sense in which ephemera like mass, color, and shape are had simpliciter. Instead, this perdurantist objects to the way in which these properties are not had simpliciter on the endurantist account. That ephemera should turn out to be relational is not itself objectionable; what's objectionable is that these relations should involve time. The thought is this: whatever properties like mass, color, and shape turn out to be, they are not inherently temporal notions. Since the endurantist claims that they are inherently temporal, endurantism is untenable.

It's unclear why time should be a particularly egregious relatum. In any case, many of these properties are inherently temporal. Given relativity, any property that varies depending on one's reference frame has a critical temporal component. This is especially easy to see with properties like spatial shape and size. Maudlin (2002) describes this well:

Take a car and a tunnel which, when at rest relative to one another, are exactly the same length. Now get in the car and drive it through the tunnel. According to the tunnel, the car is moving and therefore suffers a contraction: the car should fit entirely inside the tunnel. On the other hand, according to the car the tunnel is moving, so the car should now be longer than the tunnel. No matter how objects shrink or grow, how can it both be the case that the car is longer than the tunnel and that it is shorter than the tunnel?...

The story of the car and the tunnel illustrates how seemingly nontemporal notions may be infected by hidden temporal aspects. The question of whether the car or the tunnel is longer appears to be a question purely about the spatial qualities of the two objects... But once we see that the car being longer than the tunnel is a matter of the front and back ends of the car being outside the tunnel at the same instant, it becomes clear how the relativity of simultaneity resolves the tension between the two judgments. Since the different observers disagree on which sets of events constitute an instant, they may disagree on whether there is an instant when every part of the car is within the tunnel.

Maudlin (2002, 53-55)

Our everyday notions do not always recognize the role time plays in properties like shape or distance. But the intuition that such properties are divorced from time is mistaken, and it should not count as a mark against the endurantist that she cannot capture it. 


\section{The Fundamental Simpliciter Argument}

And yet the suspicion that there is something wrong with the endurantist's ephemera persists. Why?

For simplicity, assume that the endurantist's picture characterizes ephemera as relations between objects and times (as opposed to the adverbial variant of endurantism, which relativizes the instantiation relation). There is nothing objectionable about these relations per se. After all, the perdurantist accepts the very same relations. I am not happy simpliciter, says the perdurantist; I bear the happy-at relation to every time at which I have a happy temporal part. ${ }^{17}$ So the perdurantist worry cannot simply be that the endurantist posits relations involving objects and times.

Lewis states that the endurantist's relations to times (and the relational properties built out of them) are acceptable so long as they are "not alleged to be fundamental properties of the sort that might figure in a minimal basis on which all else supervenes." (Lewis 2002, 4) Perhaps this is the source of the worry. What's wrong with the endurantist account is not so much that it posits these relations to times, but that it posits them as fundamental. So the perdurantist objection is this: the fundamental relations simply do not include those required by the endurantist account of persistence. ${ }^{18}$

We can capture this perdurantist objection to endurantism with a third version of the Argument from Temporary Intrinsics. Call this the Fundamental Argument:

P1". Relations involving objects and times are not fundamental.

P2". If endurantism is true, relations involving objects and times are fundamental.

$\mathbf{C}^{\prime \prime}$. Endurantism is false.

While the previous versions of the Argument from Temporary Intrinsics invoked intuitions about the properties and relations involved in ordinary matters of change, this version involves a claim about the underlying properties and relations. For the endurantist, relations involving objects and times are fundamental; for the perdurantist, they are not. This version of the Temporary

\footnotetext{
${ }^{17}$ Lewis writes, "I cannot object to these relations and relational properties...I accept similar relations and relational properties myself" (Lewis 2002, 4).

${ }^{18}$ The one exception may be the occupation relation that holds between objects and the spacetime regions they occupy. The exception does not arise for the perdurantist who identifies objects with spacetime regions.
} 
Intrinsics Argument avoids the problems that beset the other two, and I believe it gets at the core worry behind the original Argument from Temporary Intrinsics.

\section{Fundamental Relations involving Objects and Times}

$\mathrm{P} 1^{\prime \prime}$ is a claim about the nature of the fundamental properties and relations. But whether there are fundamental relations involving objects and times is partly an empirical matter. As it turns out, the gauge theories of standard particle physics theories that describe the gauge properties of fundamental particles - require irreducible relations of the sort $\mathrm{P} 1^{\prime \prime}$ rules out.

We can bring out the relevant features of gauge theories by looking at how to compare gauge properties. Fortunately, we don't need to get bogged down in the details of gauge theory. Mathematically, comparing gauge properties is directly analogous to comparing the directions of vectors. So let's turn away from gauge theory for a moment, and look at how to compare the directions of vectors.

\subsection{Cannons, arrows, and quarks}

Suppose you and a friend are visiting the Fortress of Carcassonne in France. According to your guidebook, the cannon in the Northern tower points in the same direction as the cannon in the Southern tower. But your friend is skeptical; she thinks the cannons are skewed with respect to one another. How might you resolve the matter?

Unfortunately, you've lost your compass. But you notice that there is a stack of tapered boards lying nearby, where construction workers are renovating the rampart that connects the two towers. Here's a way you can determine whether the cannons point in the same direction. Go to the cannon in the Northern tower and lay down one of the boards parallel to it, one step to the south, with the tapered end pointing in the same direction as the cannon. Have your friend check to make sure you haven't twisted or turned the orientation of the board relative to the cannon. When she's satisfied, take another step towards the Southern tower, and put down a second board parallel to the first. Once your friend has checked that they're parallel, lay down a third board, and so on, until you reach the cannon in the Southern tower. Eventually, you have a straight path of boards running from one cannon to the other. Since each cannon is parallel to the board next to it, and the boards are parallel to each other, you deduce that the two cannons point in the same direction.

This situation is directly analogous to comparing the direction of vectors. To compare the directions of two vectors, we need to shift, or "parallel transport," 
one of the vectors over to the other. If we replace the cannons with vectors and the rampart with a two-dimensional plane, that's essentially what we've done in the case above.

In the example of the Fortress of Carcassonne, this was relatively straightforward. The rampart connecting the two cannons was approximately flat, so we were able to give an unequivocal answer as to whether the cannons point in the same direction simply by laying out boards in the way described. But things are not so straightforward when the surface involved isn't flat.

Consider how you might compare the directions of a pair of vectors lying on the surface of a sphere, as shown in the following diagram:



One of the vectors is located at the North Pole, and the other at the South Pole. Do these two vectors point in the same direction?

If we parallel transport the vector at the North Pole to the South Pole along path $\alpha$, as shown in the diagram below, then the two vectors will point in the same direction. But if we parallel transport the vector at the North Pole to the South Pole along path $\beta$, then the two vectors will point in opposite directions. On curved surfaces, whether the direction of one vector is the same as the direction of another depends on the path along which they are parallel transported. Two vectors on a sphere don't point in the same direction simpliciter - they point in the same direction relative to a path. 


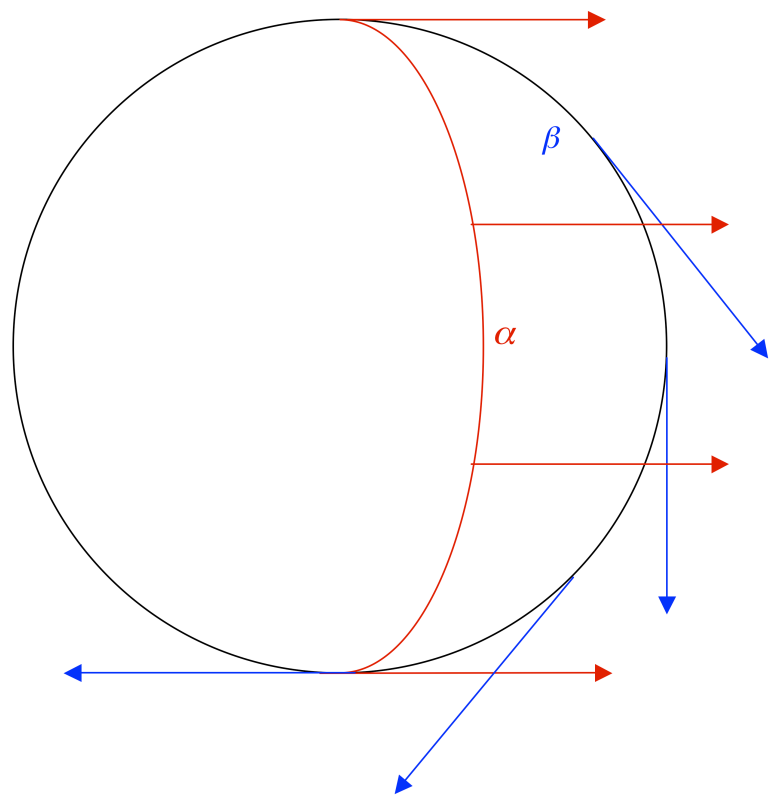

So we can see that assessing the directions of vectors in a space depends on the path through that space used compare them. In the Carcassonne example, the space is four-dimensional spacetime, so the paths involved are spatiotemporal paths. Although we compared the directions of the two cannons at the same time, we could have made any number of other comparisons, such as cross-time comparisons (e.g. comparing the direction of a cannon to itself five minutes later) or comparisons across time and space (e.g. comparing the direction of one cannon to the direction of the other five minutes later).

Now consider the gauge theory of quantum chromodynamics, according to which quark particles have one of three different "color" properties. ${ }^{19}$ The comparison of the quark colors of quarks is directly analogous to the comparison of the directions of vectors: it depends on the path along which they are compared. Two quarks don't have the same color simpliciter - they have the same color relative to a path.

What are the fundamental properties and relations that ground these facts about quark color? One natural account appeals to fundamental relations between pairs of quarks and a spatiotemporal path connecting them. Another appeals to fundamental relational properties, such as having the same color as quark q along path $p$. Or perhaps the best account of quark color will invoke fundamental relations between pairs of quarks and every spatiotemporal path

\footnotetext{
${ }^{19}$ My discussion of quantum chromodynamics draws heavily on Maudlin (2006).
} 
connecting them. But whatever account of gauge properties we ultimately settle on, the underlying gauge facts will appeal to relations involving objects and spatiotemporal paths.

Recall P1" of the Fundamental Argument: relations involving objects and times are not fundamental. But we've seen that the gauge theories of particle physics require fundamental relations between objects and paths in spacetime. $A$ fortiori, the gauge theories require fundamental relations involving objects and time. And the fact that this is so has nothing to do with the metaphysics of persistence. Since P1" is false, the Fundamental Argument is unsound.

\section{The Moral}

In the debate over the nature of persistence, our intuitions about intrinsic change are often used as the ammunition. The original Argument from Temporary Intrinsics and Lewis's Having Simpliciter version are attempts at translating these intuitions into cogent arguments. But these arguments have limited force against endurantism, since neither perdurantism nor endurantism can accommodate our pretheoretic beliefs about ephemera. A more promising way to articulate the intuitive objection behind the Temporary Intrinsics Argument is to shift the context of the argument to the fundamental properties. Yet this argument fails as well. It seems that no argument presented in the spirit of the original gives us a compelling reason to reject endurantism.

These aren't grounds to reject perdurantism. But it does suggest that if we want decisive reasons to prefer perdurantism to endurantism, we should look for them elsewhere. ${ }^{20}$

\footnotetext{
${ }^{20}$ Many thanks to John Hawthorne, Ted Sider, Kelly Trogdon, and an anonymous referee for helpful comments. Special thanks to Chris Meacham for extensive comments and discussion.
} 


\section{References}

Phillip Bricker (1993). “The Fabric of Space: Intrinsic vs. Extrinsic Distance Relations." Midwest Studies in Philosophy XVIII: 271-294.

Ben Caplan (2003). "Why So Tense about the Copula?" Mind 114: 703-708.

Hartry Field (1973). “Theory Change and the Indeterminacy of Reference.” The Journal of Philosophy 70: 462-481.

Sally Haslanger (1989). "Endurance and Temporary Intrinsics.” Analysis 49: 119125.

Mark Hinchliff (1996). “The Puzzle of Change.” Philosophical Perspectives 10, Metaphysics: 119-136.

Frank Jackson (1998). From Metaphysics to Ethics: A Defence of Conceptual Analysis. Oxford: Clarendon Press.

David Lewis (1983). "New Work for a Theory of Universals." Australasian Journal of Philosophy 61: 343-377.

David Lewis (1986). On the Plurality of Worlds. Oxford: Basil Blackwell.

David Lewis (1988). "Rearrangement of Particles: Reply to Lowe." Analysis 48: 6572 .

David Lewis (2002). “Tensing the Copula.” Mind 111: 1-13.

Tim Maudlin (2002). Quantum Non-Locality and Relativity. Oxford: Basil Blackwell.

Tim Maudlin (2006). "Suggestions from Physics for Deep Metaphysics," in Metaphysics Within Physics. Oxford: Oxford University Press.

Trenton Merricks (1994). "Endurance and Indiscernibility." The Journal of Philosophy 91: 165-184.

Bradford Skow (2007). “Are Shapes Intrinsic?” Philosophical Studies 133: 111-130.

Peter van Inwagen (1990). “Four-Dimensional Objects.” Nous 24: 245-255.

Ryan Wasserman (2003). "The Argument from Temporary Intrinsics.” Australasian Journal of Philosophy 81, pg. 413-419. 